\title{
Role of Fractional Powers in manuevering the Fractional Lower-Order Auto-Covariance of Symmetric alpha-stable noise signals
}

\author{
Areeb AHMED ${ }^{1}$ \\ Department of Electrical Engineering \\ ${ }^{1}$ Mohammad Ali Jinnah University \\ Karachi, Pakistan \\ areebahmed@iyte.com.tr
}

\author{
Ferit Acar SAVACI ${ }^{2}$, Muath WAHDAN ${ }^{2}$ \\ Department of Electrical and \\ Electronic Engineering \\ ${ }^{2}$ Izmir Institute of Technology \\ Izmir, Turkey \\ acarsavaci@iyte.com.tr, muathwahdan@iyte.edu.tr
}

\author{
Husam OTHMAN ${ }^{3}$ \\ Department of Electrical and \\ Electronic Engineering \\ ${ }^{3}$ Anadolu University \\ Eskisehir, Turkey \\ hzmo@anadolu.edu.tr
}

\begin{abstract}
Since, Fractional Lower-Order Auto-Covariance (FLOAC) remains the only technique to quantify the similarity between alpha-stable ( $\alpha$-stable) signals, therefore, the effects of impulsiveness and skewness parameters has also been analyzed before for better generation and detection in various applications. This paper includes the detailed analysis of the FLOAC of symmetric alpha-stable $(\mathrm{S} \alpha \mathrm{S})$ noise signals in order to observe the possible involvement of the associated fractional powers. The two associated fractional powers of FLOAC has been maneuvered in three possible ways to observe the probable trend of $\mathrm{S} \alpha \mathrm{S}$ noise signals in the presence and absence of Gaussian noise. The observation depicts that the fractional powers largely and solely affect the FLOAC when they are maneuvered collaboratively or even individually where the obtained results can be useful in improving many $\mathrm{SaS}$ noise signal processing techniques, especially, in the detection of $\mathrm{S} \alpha \mathrm{S}$ noise carrier signals in Random Communication Systems.
\end{abstract}

Keywords-Fractional lower-order auto-covariance; Alphastable noise; Gaussian noise; Random communication system

\section{INTRODUCTION}

Alpha-stable ( $\alpha$-stable) noise signal's detection has remained a hot topic in the past where, especially, the Fractional Lower-Order Auto-Covariance (FLOAC) has been exploited to achieve better efficiency. The $\alpha$-stable noise plays a key role in biomedical signal processing applications which involves $\alpha$ stable noise filtering and neuroimaging [1, 2]. Especially, molecular communication systems also utilize $\alpha$-stable noise as a single noise factor to mimic the effects of noise produced by several molecules in the environment [3]. Additionally, the involvement of $\alpha$-stable noise in manipulating the neural networks has also been investigated [4]. Moreover, Random Communication Systems (RCS) [5] are the example of the ongoing investigations related to $\alpha$-stable noise where these noise signals have been used as carrier signals to perform covert communications. Alpha-stable noise-based communications is an unconventional form of covert communications where the idea was initially propagated in [6].

The FLOAC has helped in proposing the first synchronization for RCSs [7, 8] where the capability of impulsive and skewness parameters to fluctuate the FLOAC was observed [9]. However, no detailed study has been carried out then to explore the role of fractional powers in maneuvering the FLOAC of $\mathrm{S} \alpha \mathrm{S}$ signals. The obtained results in this paper would benefit different $\alpha$-stable noise signal detection schemes, especially RCSs8. In the following Section-II and Section III, $\alpha$-stable noise distribution and FLOAC has been briefly explained, respectively. In Section IV, the observed results are presented where the paper has been concluded in Section-V.

\section{AlPha-StABle Distribution}

The samples of $\alpha$-stable noise can be produced via method given in [10] to generate the $\alpha$-stable distribution. The sequence of $\alpha$-stable noise, i.e. collection of consecutive $\alpha$-stable noise samples, is represented by $\mathrm{X} \sim \mathrm{S}_{\alpha}(\beta, \gamma, \mu)$ where $\alpha$ has been defined as the impulsiveness parameter in the range $(0<\alpha \leq$ 2), $\beta$ has been defined as the skewness parameter defined in the range $\beta(-1 \leq \beta \leq 1), \gamma$ has been defined as the dispersion parameter in the range $(\gamma \geq 0)$ and $\mu$ has been defined as the location parameter which is $\mu \in R$ in [11]. The characteristic function of $\mathrm{X} \sim \mathrm{S}_{\alpha}(\beta, \gamma, \mu)$ is expressed in [11] as

$$
\begin{aligned}
& \phi(\theta)= \\
& \left\{\begin{array}{c}
\exp \left\{j \mu \theta-\gamma^{\alpha}|\theta|^{\alpha}\left(1-j \beta \operatorname{sign}(\theta) \tan \left(\frac{\alpha \pi}{2}\right)\right)\right\} \text { if } \alpha \neq 1 \\
\exp \left\{j \mu \theta-\gamma|\theta|\left(1+j \beta \frac{2}{\pi} \operatorname{sign}(\theta) \ln \left(\frac{\alpha \pi}{2}\right)\right)\right\} \text { if } \alpha=1
\end{array}\right.
\end{aligned}
$$

Note: $\mathrm{X}_{\mathrm{G}} \sim \mathrm{S}_{\alpha=2}\left(\beta=0, \gamma_{G}, \mu_{G}\right), \mathrm{X}_{\mathrm{C}} \sim \mathrm{S}_{\alpha=1}\left(\beta=0, \gamma_{C}, \mu_{C}\right), \mathrm{X}_{\mathrm{L}} \sim$ $\mathrm{S}_{\alpha=0.5}\left(\beta=1, \gamma_{L}, \mu_{L}\right)$ generates Gaussian, Cauchy and Levy distributed samples, respectively, as these are special cases of $\alpha$-stable distributions.

\section{FRACTIONAL LOWER-ORDER AUTO-COVARIANCE}

Covariance and Correlation, i.e. conventional time delay estimation methods, cannot be applied for signal processing of $\alpha$-stable noise signals for $\alpha<2$ due to the absence of moments greater than second-order. Therefore, the resemblance among $\alpha$ stable noise signals can be checked by FLOAC method $[9,12]$.

If the FLOAC of $\alpha$-stable noise signal $\mathrm{X} \sim \mathrm{S}_{\alpha}(\beta, \gamma, \mu)$ is defined as 


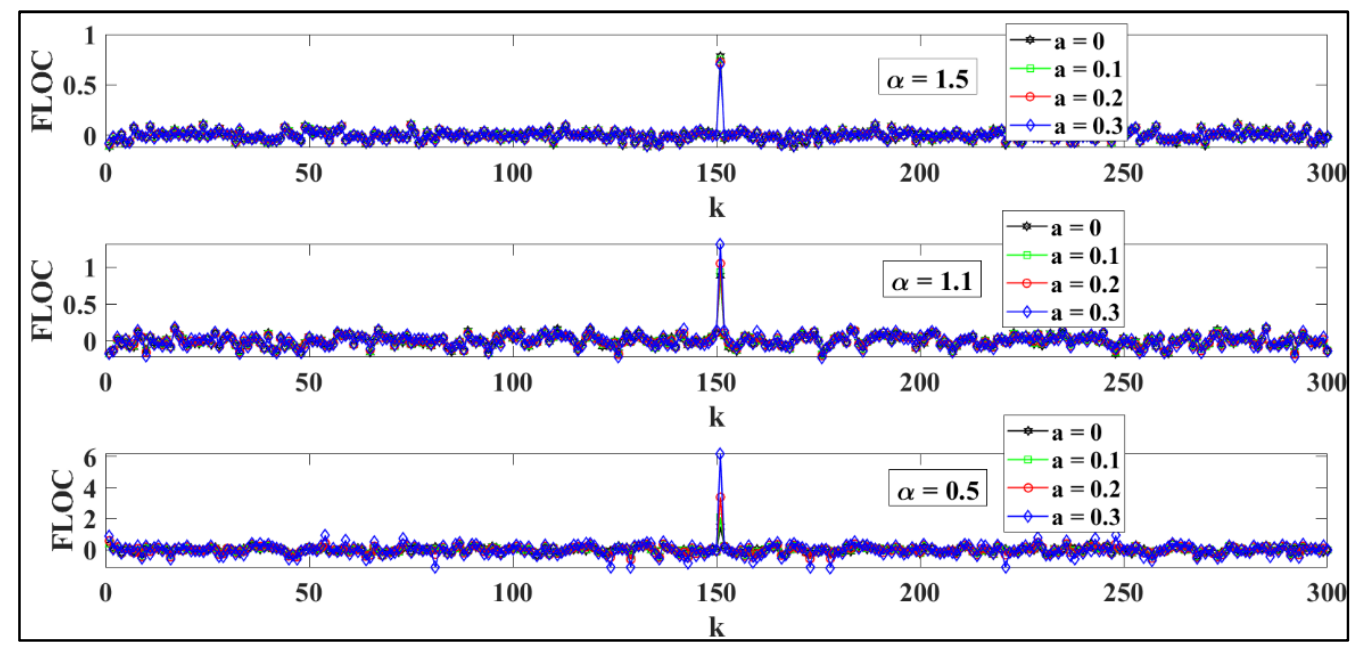

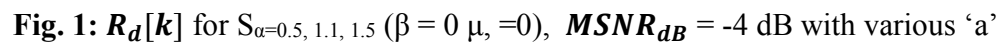

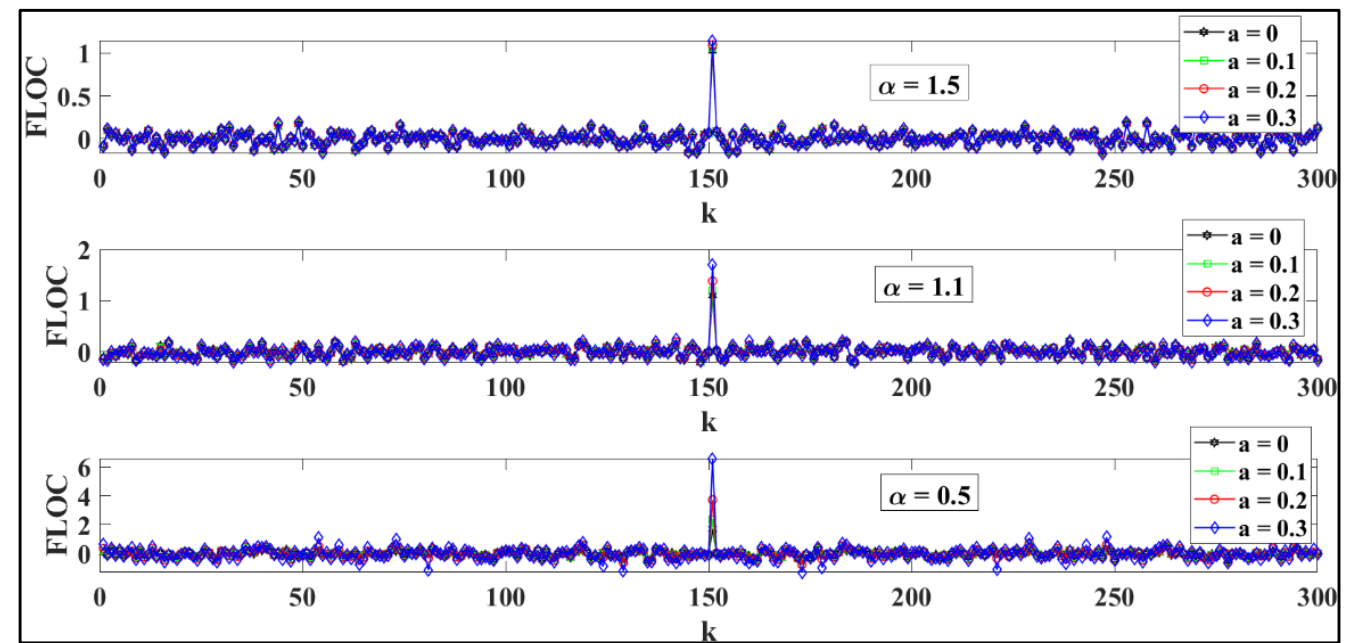

Fig. 2: $\boldsymbol{R G}_{\boldsymbol{d}}[\boldsymbol{k}]$ for $\mathrm{S}_{\alpha=0.5,1.1,1.5}(\beta=0, \mu,=0), \boldsymbol{M S N} \boldsymbol{R}_{\boldsymbol{d B}}=-4 \mathrm{~dB}$ with various ' $\mathrm{a}$ '

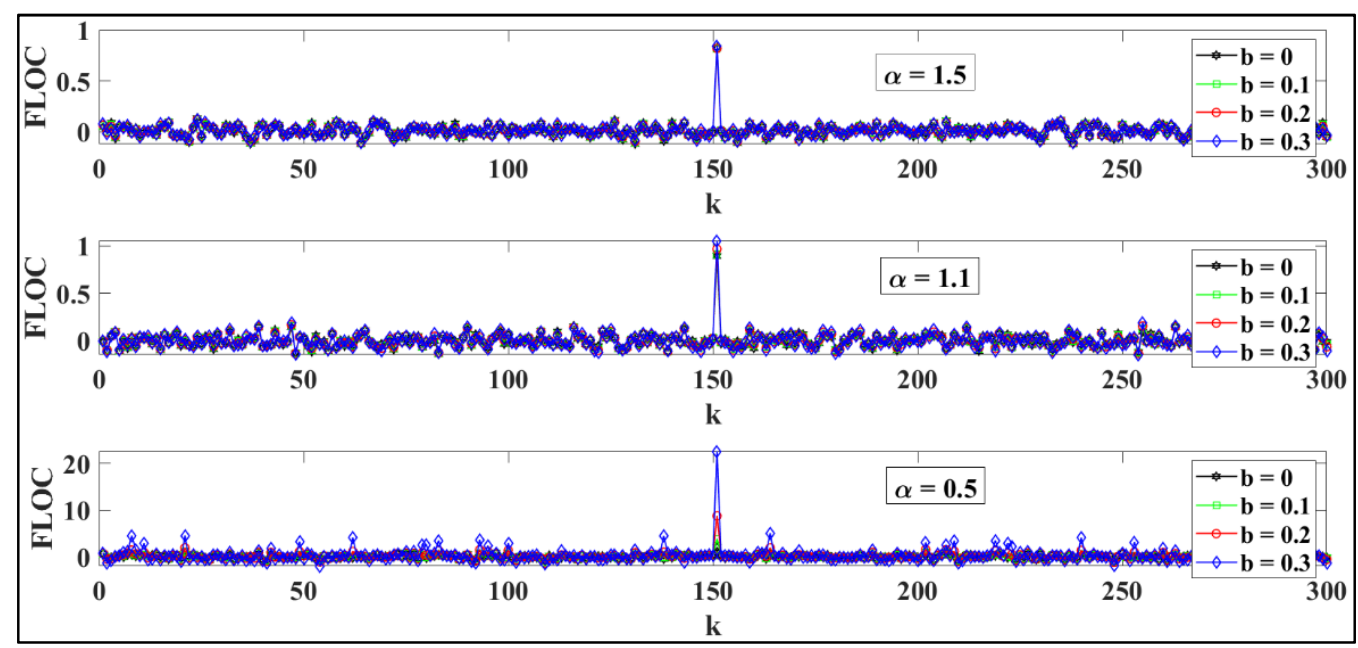

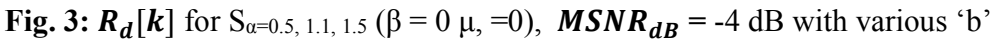




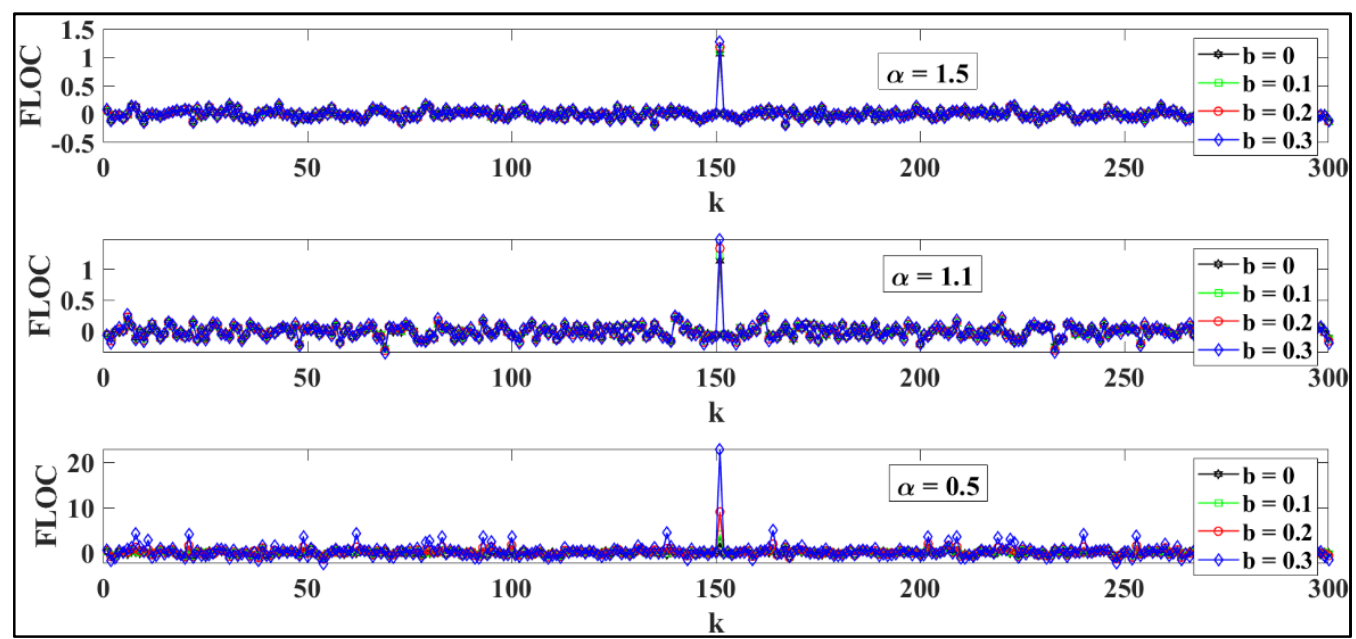

Fig. 4: $\boldsymbol{R}_{\boldsymbol{d}}[\boldsymbol{k}]$ for $\mathrm{S}_{\alpha=0.5,1.1,1.5}(\beta=0, \mu,=0), \boldsymbol{M S N} \boldsymbol{R}_{\boldsymbol{d} \boldsymbol{B}}=-4 \mathrm{~dB}$ with various 'b'

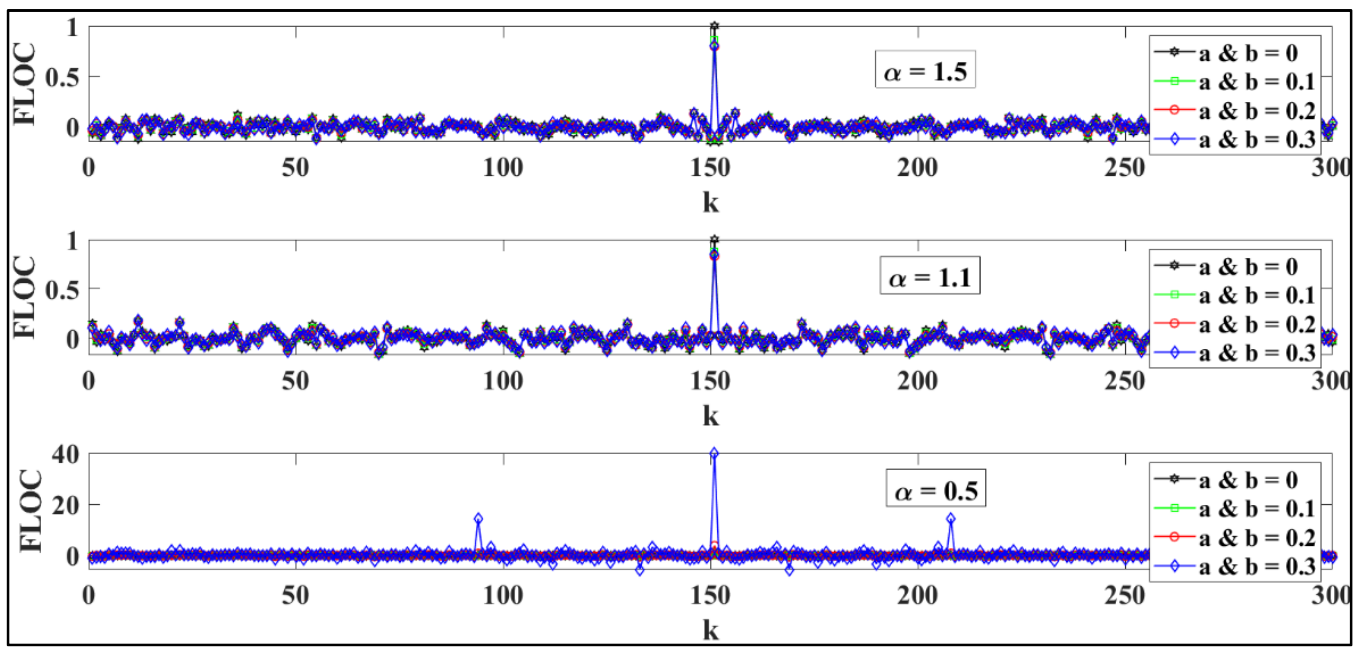

Fig. 5: $\boldsymbol{R}_{\boldsymbol{d}}[\boldsymbol{k}]$ for $\mathrm{S}_{\alpha=0.5,1.1,1.5}(\beta=0 \mu,=0), \boldsymbol{M S} \boldsymbol{N} \boldsymbol{R}_{\boldsymbol{d} \boldsymbol{B}}=-4 \mathrm{~dB}$ with various ' $\mathrm{a}=\mathrm{b}$ '

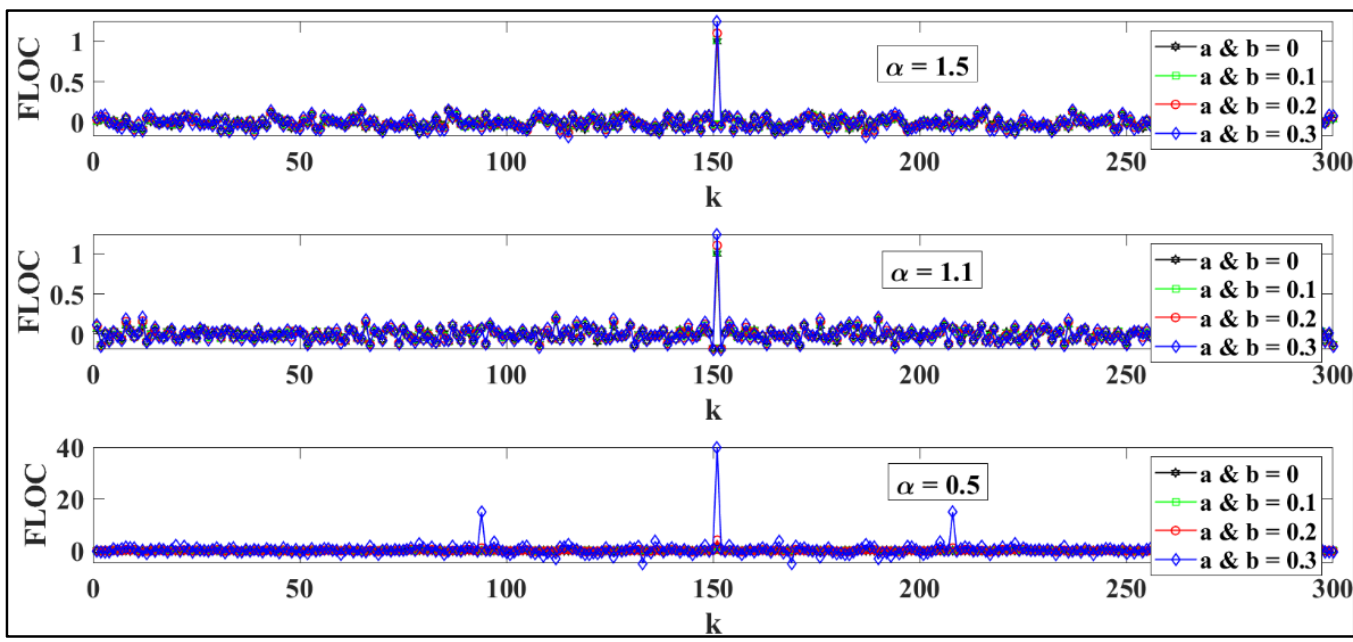

Fig. 6: $\boldsymbol{R}_{\boldsymbol{d}}[\boldsymbol{k}]$ for $\mathrm{S}_{\alpha=0.5,1.1,1.5}(\beta=0, \mu,=0), \boldsymbol{M S N}_{\boldsymbol{d B}}=-4 \mathrm{~dB}$ with various ' $\mathrm{a}=\mathrm{b}$ ' 


$$
\boldsymbol{R}_{\boldsymbol{d}}[\boldsymbol{k}] \triangleq \boldsymbol{E}\left\{(x[i])^{a} \cdot(x[i+k])^{b}\right\}
$$

then the FLOAC, denoted by $R_{d}[k]$, of $\mathrm{X}$, i.e. $\mathrm{X}=$ $\{x[1], \ldots, x[n] ; n=1,2, \ldots, N\}$, is estimated in [12] as

$$
\boldsymbol{R}_{\boldsymbol{d}}[\boldsymbol{k}]=\frac{\sum_{n=N_{1}+1}^{N_{2}}|x[n]|^{a} \cdot|x[n+k]|^{b} \cdot \operatorname{sign}(x[n] \cdot x[n+k])}{N_{2}-N_{1}}
$$

where $N_{1}=\max (0,-k), N_{2}=\min (N-k, N), k=\left(\frac{-N}{2}, \frac{N}{2}\right)$. Similarly, the noisy FLOAC, $R G_{d}[k]$, of $\alpha$-stable noise signal $\mathrm{X} \sim \mathrm{S}_{\alpha}(\beta, \gamma, \mu)$ added with $\mathrm{X}_{\mathrm{G}} \sim \mathrm{S}_{\alpha=2}\left(\beta=0, \gamma_{G}, \mu_{G}\right)$, i.e. $\mathrm{X}+$ $\mathrm{X}_{\mathrm{G}}$, has been computed as

$$
\begin{aligned}
& \boldsymbol{R G}_{\boldsymbol{d}}[\boldsymbol{k}]= \\
& \frac{\sum_{n=N_{1}+1}^{N_{2}}\left|x+x_{G}[n]\right|^{a} \cdot\left|x+x_{G}[n+k]\right|^{b} \cdot \operatorname{sign}\left(x+x_{G}[n] \cdot x+x_{G}[n+k]\right)}{N_{2}-N_{1}}
\end{aligned}
$$

However, $R_{d}[k]$ and $R G_{d}[k]$ has been computed for specific values of fractional powers, i.e. $a=b=\frac{\alpha}{2}[5,7-9,13]$, and the effects of maneuvering the fractional powers for its complete range on $R_{d}[k]$ has not been analyzed yet.

\section{ANALYSIS OF FRACTIONAL POWERS}

Therefore, in this section of the paper, the FLOACs of SaS signals, $\beta=0$ and $\mu=0$, i.e. $R_{d}[k]$ and $R G_{d}[k]$ in (3) and (4), respectively, has been computed by maneuvering ' $a$ ' and 'b' by covering entire range of $\alpha$. The FLOAC, i.e. $R_{d}[k]$ in (3), has been analyzed for three possible values of impulsiveness parameter $\alpha$, i.e. $\alpha=0.5,1.1,1.5$, to completely analyzed the behavior of FLOAC of S $\alpha$ S signals when ' $a$ ' and ' $b$ ' are varied in three possible ways, individually and collaboratively. Firstly, in Fig 1 and Fig. 2, the ' $b$ ' has been kept constant, i.e. $b=0.15$, while fractional power ' $a$ ' has been varied, i.e. $\mathrm{a}=0,0.1,0.2$, 0.3. Secondly, in Fig 3 and Fig. 4, the 'a' has been kept constant, i.e. $\mathrm{a}=0.15$, while only the fractional power ' $b$ ' has been varied, i.e. $\mathrm{b}=0,0.1,0.2,0.3$. Finally, in Fig 5 and Fig. 6 , both the fractional power ' $a$ ' and ' $b$ ' has been varied, i.e. $a=b=0,0.1$, $0.2,0.3$. The Fig. $1-6$ have been simulated by taking $N=300$ and $M S N R=-4 \mathrm{~dB}$ where the MSNR is defined in [12] as $M S N R_{d B}=10 \log \frac{\gamma}{\gamma_{G}}$ where $\gamma$ is the dispersion parameter of the $\alpha$-stable noise signal $\mathrm{X}$ and $\gamma_{G}$ is the dispersion parameter of the gaussian noise $\mathrm{G}$.

The FLOAC, i.e. $R_{d}[k]$ in (3), shows an interesting behavior as ' $a$ ' and ' $b$ ' are varied in three different ways. In the first case, the computed FLOACs $R_{d}[k]$ and $R G_{d}[k]$, in Fig. 1 and Fig. 2 , respectively, increases when ' $a$ ' increases up to the maximum limit of a, i.e. $\mathrm{a} \leq \frac{\alpha}{2}$ where $R_{d}[0]$ and $R G_{d}[0]$ is maximum when $\alpha$ is more impulsive, i.e. $\alpha=0.5$. In the second case, the computed FLOACs $R_{d}[k]$ and $R G_{d}[k]$, in Fig. 3 and Fig. 4, respectively, again increases when 'b' increases up to the maximum limit of $\mathrm{b}$, i.e. $\mathrm{b} \leq \frac{\alpha}{2}$ where $R_{d}[0]$ and $R G_{d}[0]$ is maximum when $\alpha$ is more impulsive, i.e. $\alpha=0.5$. In the third case, the computed FLOACs $R_{d}[k]$ and $R G_{d}[k]$, in Fig. 5 and Fig. 6, respectively, increases when ' $a$ ' and ' $b$ ' increases up to the maximum limit of $\mathrm{a}$ and $\mathrm{b}$, i.e. $\mathrm{a}=\mathrm{b} \leq \frac{\alpha}{2}$ where $R_{d}[0]$ and $R G_{d}[0]$ is maximum when $\alpha$ is more impulsive, i.e. $\alpha=0.5$.

It has been seen that $R_{d}[0]$ and $R G_{d}[0]$ is highest among $R_{d}[k]$ and $R G_{d}[k]$ irrelevant which value of fractional power and impulsiveness parameter has been used and it is inversely proportional to $\alpha$.

\section{CONCLUSION}

Fractional lower-order auto-covariance of $\mathrm{S} \alpha \mathrm{S}$ signals has been analyzed while maneuvering the fractional powers. It has been concluded that FLOAC of $\mathrm{S} \alpha \mathrm{S}$ results in maximum value if higher values of fractional powers and lower values of impulsiveness parameter are utilized and vice versa. The above results would be useful in enhancing the detection process of $\alpha$ stable noise signal processing techniques associated with biomedical and communications applications.

\section{REFERENCES}

[1] D. Salas-Gonzalez et. al, "Why using the alpha-stable distribution in neuroimage?," in IEEE International Conference on Signal Processing and Multimedia Applications (SIGMAP), 2014, pp. 297-301.

[2] T. Pander, "Impulsive noise filtering in biomedical signals with application of new myriad filter," in 20th biennial international EURASIP conference biosignal, 2010, pp. 94-101.

[3] N. Farsad, W. Guo and C.B. Chae, "Stable distributions as noise models for molecular communication," in Global Communications Conference (GLOBECOM), 2015, pp. 1-6.

[4] B. Kosko and S. Mitaim, "Stochastic resonance in noisy threshold neurons," Neural networks, vol. 16, no. 5, pp. 755-761, 2010.

[5] A. Ahmed and F.A Savaci, "Random Communication System Based on Skewed Alpha-Stable Levy Noise Shift Keying," Fluctuation and Noise Letters, vol. 16, no. 3, 1750024, 2017.

[6] M.E. Cek, "Covert communication using skewed $\alpha$-stable distributions," IET Electronics Letters, vol. 51, no. 1, pp. 116-118, 2015.

[7] A. Ahmed and F.A Savaci, "Synchronization of alpha-stable Levy noise based random communication system," IET comunications, vol. 12, no. 3, pp.276-282. 2017.

[8] A. Ahmed and F.A Savaci, "On optimizing fractional lower order covariance based synchronization method for random communication systems," in 26th Signal Processing and Communications Applications Conference (SIU), 2018, pp. 1-4..

[9] A. Ahmed and F.A Savaci, "Structure and Performance Evaluation of Fractional Lower-Order Covariance Method in Alpha-Stable Noise Environments," Recent Advances in Electrical \& Electronic Engineering (Formerly Recent Patents on Electrical \& Electronic Engineering), vol. 12, no.1, pp.40-44, 2019.

[10] A. Janicki and A. Weron, Simulation and chaotic behavior of a-stable stochastic processes, C.\&Hall/CRC, 1994.

[11] G. Samorodnitsky and M. Taqqu, Stable non-gaussian random processes: stochastic models with infinite variance, C.\&Hall/CRC, 1994

[12] X. Ma and C.L. Nikias, "Joint estimation of time delay and frequency delay in impulsive noise using fractional lower order statistics," IEEE Transactions on Signal Processing, vol. 44, no.11, pp. 2669-2687, 1996.

[13] W. Liu et. al, "Evoked potential latency delay Estimation by using covariation correlation approach," in 2nd International Conference on Bioinformatics and Biomedical Engineering (ICBBE), 2008, pp. 652-655. 